\title{
Application of Tweezers Raman Spectroscopy in Microbial Research
}

\author{
Chunjuan Fang
}

School of nursing, Jiangxi University of Technology

\begin{abstract}
Keywords: Laser tweezers Raman spectroscopy; Microbiology; Rhodosporidium; Carotenoid; Spore germination.
\end{abstract}

\begin{abstract}
Laser tweezers Raman spectroscopy (LTRS) is the technology combining optical tweezers microprobe Raman spectroscopy to capture and fix the single cell in solution for a long time. It researches the real-time biochemistry changed information of the single cell covered beneath group average information under nearly natural physiological conditions. It has a series advantages in the research field of microbiology including low-energy light beam with no hurt to cells, no addition of chemical reagent and the high sensitivity of the optical technology without damaging cells. Laser tweezers Raman spectroscopy can reflect the activity law of biomacromolecule in microbiology cells, keep real-time tracking of biochemical kinetic process of microbiology and provide new technology for basic microbiology and fermentation microbiology. Therefore, the experiment is designed in the thesis by taking advantages of laser tweezers Raman spectroscopy to provide more theoretical basis and reference for the application and development of the technology.
\end{abstract}

\section{Introduction}

Microbiology is a subject researching the morphological structure, physiology and biochemistry of microbiology, and the law and application of its evolvement, classification and zoology. The introduction of new technology and methods is the key to the development of microbiology. Fluorescence labeling technique, cell membrane electrophysiological technique and high resolution microscope have made it possible for us to directly explore the life activities of the microbiology, but the antibodies and staining technique are not only costly and can also cause different levels of effects or hurt to the microbiology cell under physiological conditions. Besides, most of the technologies may involve a complex experiment operation and consume much time. Therefore, the development and application of laser tweezers Raman spectroscopy has provided a new method for microbiology research.

Raman spectrum is an inelastic scattering spectrum produced from molecule or photons at lattice vibration energy level. The position, intensity and line width of the characteristic of Raman spectrum can provide information on molecule vibration and rotation and reflect different chemical bond and functional group. Raman spectrum is a useful tool for analyzing molecule structure and contents, and now is more and more being used in the research on the cell structure, chemical compositions and metabolic process of microbiology.

The generation and principle of laser tweezers, laser Raman spectroscopy system composition and working principle. Laser tweezers Raman spectroscopy (LTRS) is a technology combining optical tweezers and microscopic Raman spectra technique. Optical tweezers is a 3D optical potential 
formed by a association of highly focused laser microbeam which can capture and fix the single cell in solution for a long time to reduce the disturbance of cell activity and Brownian movement, and thus analyze single cell under nearly natural conditions. Meanwhile, it can reduce the intensity of the background signal and fluorescence, and improve SNR of Raman spectra. Compared with other microbiology test technology, laser tweezers Raman spectrum has the advantages of non-invasion, situ real-time study and high sensitivity.

In combination with the development of Raman spectrum technology, the principle and characters of confocal microscopic Raman spectra, resonance Raman spectroscopy, surface enhanced Raman spectra, Raman imaging, coherent anti-stokes Raman spectrometry and Raman-FSH, with focus on concluding and analyzing the application advantages of different Raman spectrum technology in microbiology structure, chemical compositions and metabolic process. Therefore, there can be seen huge values of in reasonable application of these technologies in basic microbiology, fermentation microbiology, and microbiology diagnosis.

\section{Raman spectrum}

When the lights shines on the gas, liquid and the transparent crystal samples, most of the light transmits through, and only a small fraction is scatters at different angles, the phenomenon called light scattering. Analyzed from the views of quantum mechanics, a photon rises a molecular energy from ground state to virtual state - an unstable status, the molecule will immediately transmits a photon and return to the original electronic state. If the molecule can return to its original vibration energy level, the photon it transmits has the same energy as the incident photon, that is with the same wave length; while in case of no energy transmitting to the molecule, there will be Associationleigh scattering; in case the molecule returns to a higher or lower energy level, the emitting photon will have different energy, the wave length will change, and so will the vibration energy of the molecule---that is so called Raman scattering. In that case, what is increased to molecule vibration energy is stokes Raman scattering; while in an opposite case, it will be anti-stokes Raman scattering increased to the molecule vibration energy.

Under thermal balance status, the molecule number ratio at different energy levels is subject to boltzmann distribution, the number of molecules at low vibration energy level is always higher than that at higher level. Therefore, stokes Raman intensity is always stronger than anti-stokes Raman intensity, and we are mainly collecting stokes Raman spectrum.

The function diagram of the relative wave length of Raman scattering intensity is generally called Raman spectrum diagram. The common unit of the horizontal axis of Raman spectrum is the wave number relative to wavelength deviation of the exciting light (wave number is the reciprocal of the wavelength) or called Raman shift for short at the unit of $\mathrm{cm}^{-1}$. The Raman shift with the given vibration is the measurement of the vibration energy and has no relation to the laser excitation wavelength in use.

$$
I_{R}=\frac{2{ }^{4} x^{2}}{45 \times 3{ }^{2} c^{4}} \times \frac{h_{\mathrm{f}} N\left(v_{0}-v\right)^{4}}{\omega\left(1-\alpha^{-\frac{h v}{K t}}\right)}\left[45\left(\alpha_{\alpha}^{\prime}\right)^{p}+7\left(\gamma_{\alpha}^{\prime}\right)\right]
$$


Raman scattering intensity is in direct proportion to the number of photons under the exciting light. This is the basis of using Raman spectrum technology for quantitative analysis. The intensity of the Raman scattering is also in direct proportion to the intensity of the incident light. Both of the direct ratio relations are the basis for quantitative analysis by use of Raman spectrum technology.

\section{The development of Raman spectrum technology and its application in microbiology}

Early Raman spectrometer has arc lamp as the light source, and the application is quite under limits for the weak Raman scattering spectral line from the samples. That deficiency was solved only until 1960s when laser light source was born. The improvement of weak signal test technology and the application of computer has overcome the weakness of early Raman spectrum technology and made it widely used as a analytical test instrument for appraising substance structure. Subsequently, the invention of a series of new technology, like confocal Raman microspectroscopy, enhanced Raman spectroscopy, Raman imaging, also provide a broad prospect for its future application.

Confocal Raman microspectroscopy (CRM). CRM is an applied technology combining confocal optical microscopy with Raman spectrum. The confocal micro Raman system uses microobjective to focus laser beam on samples to reduce the disturbance of ambient substances and acquire accurate Raman spectrum information in local area, meanwhile, it can also observe Raman signal at different depths by changing focal depth. Compared with traditional Raman spectrum technology, confocal Raman micro-spectroscopy has great horizontal and axial resolutions, making it able to reflect the multiple-dimensional information of the chemical substances in single cell. Christian K observed some cell mutation (an increased volume) and the gathering of bacteria polysaccharides starch grains, while detecting the physiological conditions of single Bayesian clostridium through confocal microprobe Raman. There are $481^{\mathrm{cm}^{-1}}, 941^{\mathrm{cm}^{-1}}$ and 1 080 1130 ${ }^{\mathrm{cm}^{-1}}$ peaks in the Raman spectrum of the mutated cell. These peaks are all signal peaks of Bacteria starch granules. At the same time, compared with normal cells, PHE has a weaker 1 $005^{\mathrm{cm}^{-1}}$ peak and $1220 \sim 1290^{\mathrm{cm}^{-1}}$ peak of cool amine III gradually disappears, indicating the reduction of related protein. $1050^{-1}$ peak of the saccharides remains the same level, making the substance likely one of the constituents of cell wall. Confocal microprobe Raman spectroscopy can also research phenotypic heterogeneity of the microbial population on the single-cell level. Hermelink A proved by use of confocal microprobe Raman spectroscopy that even the bacterial colony cultivated under laboratory conditions also has spectrum difference, showing a clear heterogeneity.

Enhanced Raman spectroscopy. Due to the low intensity of Raman scattering effect, there is inevitably trouble in low concentration and microsample test and analysis. Enhanced Raman spectroscopy can effectively overcome this deficiency. Based on the enhancement principle, the technology can be divided into two types: resonance Raman spectroscopy and surface enhanced Raman spectra.

Resonance Raman Spectroscopy (RRS) happens when the wavelength of exciting light is equal to electronic transition wavelength of molecule, with the intensity $10^{6}$ times stronger than regular 
scattering, and the greatly sharpened sensitivity can also guarantee more spectrum information than regular Raman spectrum. It is suitable for biomacromolecule sample test, since the electronic absorption area of many biomolecules are out of UV, and UV RRS has achieved remarkable effects in the research on protein, nuclear acid and filamentous virus particles. At deep $244 \mathrm{~m} \mathrm{UV}$, it can selectively strengthen the signals of aromatic amino acid and nuclear acid, andNeugebauer $U$ can use deep UV RRS to research the growth process of bacillus and the anti-drug of antibody Ciprofloxacin. When it uses $24 \mathrm{~nm}$ exciting light, it can be discovered the blut shift and strengthen decline of 1480 $\mathrm{cm}^{-1}$ peak of the representing nuclear acid during the lag period log, phase and stability period of the bacterial, $1607^{\mathrm{cm}^{-1}}$ peak of the representation protein strengthens, meanwhile, it suggests that deep UV RRS is suitable for detecting DNA and protein change. By comparing the Raman spectrums of the control cells with different doses of Ciprofloxacin $(0.9 \mathrm{ug} / \mathrm{rnL}, 5.0 \mathrm{ug} / \mathrm{mL})$, they also discovered that the near areas of $1480^{-1}$ and $1620^{-1}$ peaks changed and showed an obvious differences. In combination with clustering analysis and the major composition analysis, it can be easier to differentiate the cells treated under different doses of anti-body. The method can be used for bacterial anti-drug attribute research. Because of the high energy of the UV wavelength used by RRS, its heat effect and photochemical effect may damage the samples, however, the effects can be reduced by shortening the integral time and lowering the laser strength.

\section{Laser tweezers Raman spectroscopy}

In 1970s, Zhu Diwen for the first time invented laser cooling and trapped atoms method by use of photo-pressure principle. Ever since, people began to use the principle to explore the mechanical effects of light on the tiny macroparticle. In 1986, Ashkin A formed an optical potential with stable three dimensions by a association of highly concentrated laser, which can trap biological particles in the potential to capture and shift the biological particles. That was how the so called laser tweezers were invented. Optical tweezers is a new physical tool concerning tiny macroparticles based on the mechanical effects of light, which can realize non-destructive capturing and operation of cell and cell wall.

Optical tweezers is a kind of 3D optical potential formed by the single association of laser emitted from laser device after highly focusing under the microscope with high-expansion aperture. Assume a highly focused laser shines onto a transparent ball particle in liquid, part of the light will be reflected at the boundary between the liquid and the particle, and another part reflected into the particle, since the reflection rate of the particle is bigger that the ambient liquid. The reflection will change transmission direction of the light on the surface of the particle, and thus change the momentum direction acting upon the particle. Based on momentum conservation law, the particle will get the momentum from reverse direction to keep its total momentum unchanged. According to Newton second law, the acting force that the object receives is equal to the change amount of the momentum within unit time. Therefore, we can assume the acting force the particle imposes onto the photon is $F_{A}$ meanwhile, according to Newton third law which says any acting force has an equal counter-acting force in reverse direction, so the particle also receives a counter-acting force $F_{A}$ 
from the photon which can be called gradient force directing to the point with the biggest light intensity, in this way a 3D optical potential---optical tweezers forms at the focus.

\section{Technical advantage and application of laser tweezers Raman spectroscopy}

Laser tweezers Raman spectroscopy is the technology combining optical tweezers and microprobe Raman spectroscopy to capture and fix the small single cell in solution for a long time. It can reduce the disturbance of cell movement and Brown movement and research the real-time biochemistry change information of the single cell covered beneath group average information under nearly natural physiological conditions without having add any chemical drug and damaging cell with the low-energy beam. Because the particle is fixed near the convergent focus of the laser beam, the optical collection route of the scattering light can be improved to enhance SNR of the spectrum signal.

The substances changes in quite a complex way in cell metabolic process. To make it more suitable for cytology research, Raman spectrum detection technology has been combined with multiple cell research instruments to derive many branch technologies. Laser tweezers Raman spectroscopy can monitor single cell kinetic process by real time and holds a huge potential in exploring microbiology life rule. Chen D ever made real-time monitoring thuringiensis bacillus spores in TSB nutrient solution through LTRS. From the real-time Raman spectrum information, it was drawn that 2,6- $\mathrm{Ca}^{2+}$-DPA changes with time, and that discovery can be sued for tracking the process of spire germination. The research team has also researched complex kinetic process of single cell disruption of escherichia coli. In the process, the intensity of the peaks $668^{\mathrm{cm}^{-1}}$, $725^{\mathrm{cm}^{-1}}, 783 \mathrm{~cm}^{-1}, 813^{\mathrm{cm}^{-1}}, 1100^{\mathrm{cm}^{-1}}, 1480^{\mathrm{cm}^{-1}}, 1574^{\mathrm{cm}^{-1}}$ of the nuclear acid gradually declines, which suggest that RNA related substances within the cell are released outside out the damaged cell. In the process of gentle phage induction at moderate temperature, the intensity of the peaks $813^{\mathrm{cm}^{-1}}, 1574^{\mathrm{cm}^{-1}}$ and other related peaks (except $783^{\mathrm{cm}^{-1}}$ and $1100^{\mathrm{cm}^{-1}}$ ) of the nuclear acid begin to rise again after a slightly drop. The drop of the peaks is perhaps because of the part decomposition of ribosome or the disappearance of RNA in ribosome and other RBA double-chain structure in cell; the subsequent rose may be because the combination of phage DNA and protein increase the number of RNA in ribosome, which is verified by stable status of $783,1100^{-1}$ peak unrelated to RNA structure. LTRS can reflect entirely different changes in the two disruption processes and serve as the powerful tool monitoring the complex dynamic change in the cell.

\section{LTRS monitoring the real-time germination of yeast spore}

Spore, otherwise known as endospore and germ, is a hypopus formed under a very unfavorable growth environment for bacterial, when the nutrient cell stops growing and splitting and cytoplasm contracts for serious dehydration. In dormancy status, spore almost stops metabolic process, and can resist any external unfavorable elements, including high pressure, heat and humidity, dry, extreme $\mathrm{PH}$ value and some toxic chemical substances. Despite the dormancy status, it is extremely sensitive 
to ambient environment, and once detects favorable living environment, it can continue to absorb the nutrients around to grow up to be nutrient cell, by that time, the extreme endurance capacity also disappears.

LTRS has advantages in research the single cell or organelle in solution nearly its physiological conditions. The real-time average Raman spectrum of single cell can display the accumulative change of the substances in cell in the process of spore germination: the peaks $722^{\mathrm{cm}^{-1}}, 1$ $006^{\mathrm{cm}^{-1}}$ of DNA and protein are first unchanged and then rise up during germination period, which suggests that DNA has begun to duplicate and synthesize to protein at large scale in the cell during that period; while the peak $1751^{\mathrm{cm}^{-1}}$ of the lipid dramatically drops perhaps because of lipid substance consumption. During the whole period of germination, the intensity of the peaks 858 $\mathrm{cm}^{-1}, 908^{\mathrm{cm}^{-1}} 084^{\mathrm{cm}^{-1}}, 1118^{\mathrm{cm}^{-1}}$ originated from glucose or mycose rise after a decline, suggesting that under appropriate living conditions, mycose is lively to convert to glucose to be absorbed by cell, and when nutrients are gradually consumed, the cell will accumulate mycose once again to resist external conditions. According to the result, it can be summarized that LTRS can take real-time monitoring of yeast spore germination biochemical kinetics process, reflect the activity rule of biomacromolecule, acquire rich information on material change in the process of yeast spore germination. The technology is a very practical tool for exploring the real-time biochemical change of single living cell and can provide new method for basic microbiology research.

\section{Conclusions}

The thesis has first introduced Rama spectrum generation principle, concluded and analyzed the application advantages of the different Raman spectroscopyies in the research on microbiological structures, chemical compositions and metabolic process. Laser tweezers Raman spectroscopy is the technology combining optical tweezers microprobe Raman spectroscopy to capture and fix the single cell in solution for a long time. It researches the real-time biochemistry changed information of the single cell covered beneath group average information under nearly natural physiological conditions. It has a series advantages in the research field of microbiology including high efficiency and sensitivity, no chemical medicines, no damage to cells and low-energy light beam without hurt to cells. The thesis aims to design a detailed experiment scheme to explore the feasibility and application value of the approach.

Laser tweezers Raman spectroscopy is a new technology in researching fermentation microbiology, which can effectively monitor the dynastic process of microbiology fermentation, acquisition of base consumption, product generation and the change of other compositions in the cell. The technology can reflect the activity rule of biomacromolecule in microbiology cell, keep track of microbiology biochemical kinetics by real time and provide basis for basic microbiology research. Since the technology can be applied to single bacterial, clinical samples can be directly analyzed without having to cultivate the bacterial. With efficient integration of multiple technologies, Laser tweezers Raman spectroscopy will play increasingly important role in microbiology diagnosis, physiology and biochemistry research and medical experiment. 


\section{References}

[1]Shen Ping, Chen Xiangdong. Microbiology [M]. Beijing: Higher Education Press, 2006:9

[2]Tao Zhanhua, Yao Huilu, Wang Guiwen, etc. Using Raman Spectrum to Analyze Gastric Cancer Apoptosis Induced by Cisplatin [J]. Spectroscopy and Spectral Analysis，2009, 29(9):2441-2445

[3] Tao Zhanhua, Wang Guiwen, Xu Xiaodong, et ah Monitoring and rapid quantification of total carotenoids in Rhodotorulaglutinis cellsusing laser tweezersRaman spectroscopy J]. FEMS Microbiol Lett, 2010, 314 (2011): 42-48

[4]Buijtels P C, Willemse-Erix H F, Petit P L, et al. Rapid identification of mycobacteria byRaman spectroscopy[J]. Clin Microbiol， 2008， 46(3): 961-965

[5] Papaioannou E H, Liakopoulou-Kyriakides M, Christofilos D, et.al. Raman spectroscopy forintracellular monitoring of carotenoid in Blakeslea trispora[J]. Appl Biochem Biotechnol, 2009,159(2):478-487

[6] Palchaudhuri S, Rehse SJ, Hamasha K, et al, Raman spectroscopy of xylitol uptake and metabolism in Gram-positive and Gram-negative bacteria [J]. Appl Environ Microbiol. 2011, 77(1): $131-137$

[7] Couling V W5 Fischer P, Klenerman D, et al. Ultraviolet resonance Raman study of drug binding in dihydrofolate reductase, gyrase, and catechol O- methyltransferase[J]. Biophys, $1998,75,1097-1106$

[8] El-Mashtoly S F, Gu Y, Yoshimura H, et al. Protein conformation changes of HemAT-Bs upon ligand binding probed byultraviolet resonance Raman spectroscopy[J]. Biol Chem, 2008, 283(11): 6942-6949

[9]Xing Dahui, Pan Anlong, Xue Donghua. Comparison on the Application of Different Carbon Source by Mortierella Isabellina to Produce Grease [J]. Chinese Journal of Biotechnology, 2010, 26(2): 189-193

[10]Li Yonghong, Liu Bo, Zhao Zongbao, etc. Optimization Study on Grease Medium Cultivation by Rhodosporidium Toruloides Fermentation and Fermentation Conditions [J] Chinese Journal of Biotechnology, 2006, 22(4): 650-656 